\title{
Simulation der Logistik auf Erdbaustellen
}

\author{
Dipl.-Ing. Johannes Wimmer, Prof. Dr.-Ing. Dipl.-Wi.-Ing. Willibald A. Günthner \\ Technische Universität München \\ Lehrstuhl für Fördertechnik Materialfluss Logistik
}

\begin{abstract}
Die Planung von Erdbaumaßnahmen stellt eine komplexe Aufgabe dar. Der Einsatz unterschiedlicher Maschinenkonfigurationen sowie alternativer Szenarien im Baustellenlayout (z.B. bezüglich der Transportrouten oder Zwischenlager) ist durchgängig zu prüfen und auszulegen. Falsche Entscheidungen können zu Verzögerungen oder einer unwirtschaftlichen Lösung führen und somit Projektdauer und -kosten beeinträchtigen. In der Praxis beruht dieser Planungsprozess auf dem Erfahrungswissen der Verantwortlichen, Tools zur Entscheidungsunterstützung in der Einsatzplanung von Aushub- und Transportgeräten werden entgegen der zentralen Bedeutung aber bislang nicht genutzt. Deshalb wurde im Rahmen des Forschungsverbundes „ForBAU - Virtuelle Baustelle" ${ }^{\text {11 }}$ ein Lösungsweg erarbeitet, mit dem die Planung der Baustellenabläufe im Erdbau durch die Anwendung der ereignisorientierten Ablaufsimulation unterstützt werden kann. Hierzu wurden die Verfahren der Leistungsberechnung von Erdbaugeräten um statistische Komponenten ergänzt, für eine Anwendung in der Simulation adaptiert und in eine Bausteinbibliothek implementiert. Die Ermittlung der Transportzeiten basiert in der vorgestellten Lösung auf einem eigens entwickelten Algorithmus zur kinematischen Simulation der Fahrzeiten, welcher die Beschleunigungs- und Bremsvorgänge eines Fahrzeugs auf der Wegstrecke unter Berücksichtigung der Beladung nachbildet.
\end{abstract}

\section{$1 \quad$ Einleitung}

Erdbaustellen sind vielfältigen Einflüssen und sich ändernden Randbedingungen aus gesetzt, welche zwangsläufig zu fortwährenden Anpassungen des Bauablaufs während der Ausführung führen. Die Ablaufplanung ist deshalb ein dynamischer Prozess der aufgrund der Schnelllebigkeit der Baustelle nur sehr schwer beherrschbar ist. Für einen wirtschaftlichen Betrieb ist daher ein effizienter und unter den Einzelvorgängen abgestimmter Ablauf Voraussetzung. In diesem Zusammenhang sind eine Vielzahl einzelner Vorgänge zeitlich und kapazitativ aufeinander abzustimmen. Eine Möglichkeit die dynamischen Abläufe auf der Baustelle besser abzubilden bietet die ereignisorientierte Ablaufsimulation. Jedoch erschweren die komplexen Randbedingungen auf der Baustelle die Modellierung der Bauprozesse.

Unsicherheiten, wie die Witterung, sind bisher noch nicht näher untersucht und erschweren die Modellbildung, da diese einen direkten Einfluss auf die jeweilige Arbeitsleistung haben. Bisher werden diese nur über pauschale

\footnotetext{
${ }^{1}$ Der Forschungsverbund „Virtuelle Baustelle - ForBAU“ wird gefördert durch die Bayerische Forschungsstiftung.
} 
Abminderungsfaktoren oder Durchschnittsleistungen berücksichtigt. Aufgrund von Störungen, oder unerwarteter Verzögerungen kann der ursprünglich geplante Bauablauf nicht eingehalten werden. Um teure unproduktive Zeiten zu vermeiden, wird dieser oft spontan auf Basis der aktuellen Situation umgestellt. Diese flexible Vorgehensweise muss auch in der Simulation abgebildet werden. Eine weitere Schwierigkeit bei der Modellierung von Bauabläufen sind die meist fehlenden oder schwer zugänglichen Eingangsdaten. Einerseits sind aufgrund des weitgehend unbekannten Verlaufs der Bodenschichten die zu verarbeitenden Erdmassen oftmals nur unzureichend bekannt. Andererseits sind Daten über die Lage von Bauwerk, Transportwegen und Baustelleneinrichtung in der Regel nur in Form von 2D-Plänen vorhanden, so dass diese für jede Baustelle erneut manuell in die Simulation übertragen werden müssen.

\section{Anforderungsanalyse}

Um die Anforderungen an die Ablaufsimulation und deren Ziele im Erd- und Tiefbau zu ermitteln, wurde in einem ersten Schritt ein Workshop mit den Industriepartnern des Forschungsverbundes durchgeführt. Primäres Ziel ist die simulative Absicherung von Baumaßnahmen. Um die aus den vielfältigen Einflüssen und sich ändernden Randbedingungen entstehenden Unsicherheiten in der Ablaufplanung abbilden zu können, wird eine Methode gesucht, verschiedene Szenarien vor der Bauausführung durchzuspielen und deren relevante Parameter vergleichen zu können. Ein weiteres Ziel ist die Optimierung des Maschineneinsatzes, um die Wirtschaftlichkeit einer Baustelle zu verbessern. Neben den wirtschaftlichen Aspekten ist die anschauliche Visualisierung der Baustellenabläufe in der Simulationsumgebung ein essentieller Punkt. Für die Vielzahl der Beteiligten bietet die dreidimensionale Animation des Baustellenablaufs eine übersichtliche Darstellung der konkreten Planungen, so dass Fehler aufgrund von Missverständnissen vermieden werden können.

Der Einsatz der Simulation im Erdbau eignet sich vor allem in zwei Phasen. Zum Einen in der Angebotserstellungsphase, in der innerhalb eines kurzen Zeitraums der Bauablauf erstellt und die Kosten dafür kalkuliert werden müssen. Zum Anderen sollen in der anschließenden Arbeitsvorbereitung verschiedene Szenarien miteinander verglichen werden, um gesicherte Ablaufplanungen mit hohem Detaillierungsgrad zu generieren. Daher ist es sinnvoll, ein spezifisches Simulationsmodell für ein festgelegtes Bauprojekt zu erstellen, welches von der relativ groben Planung in der Angebotskalkulation bis zur Feinplanung in der Arbeitsvorbereitung durchgängig genutzt werden kann. Dabei sollten die in Abbildung 1 dargestellten Anforderungen erfüllt werden.

Für eine gesicherte Ablaufplanung von Erdbauvorhaben müssen mehrere Szenarien miteinander verglichen werden. In der Ausführung ergeben sich vielfältige Variationsmöglichkeiten, welche unabhängig voneinander veränderbar sein müssen, um flexibel auf die jeweiligen Baustellenrandbedingungen reagieren zu können. 


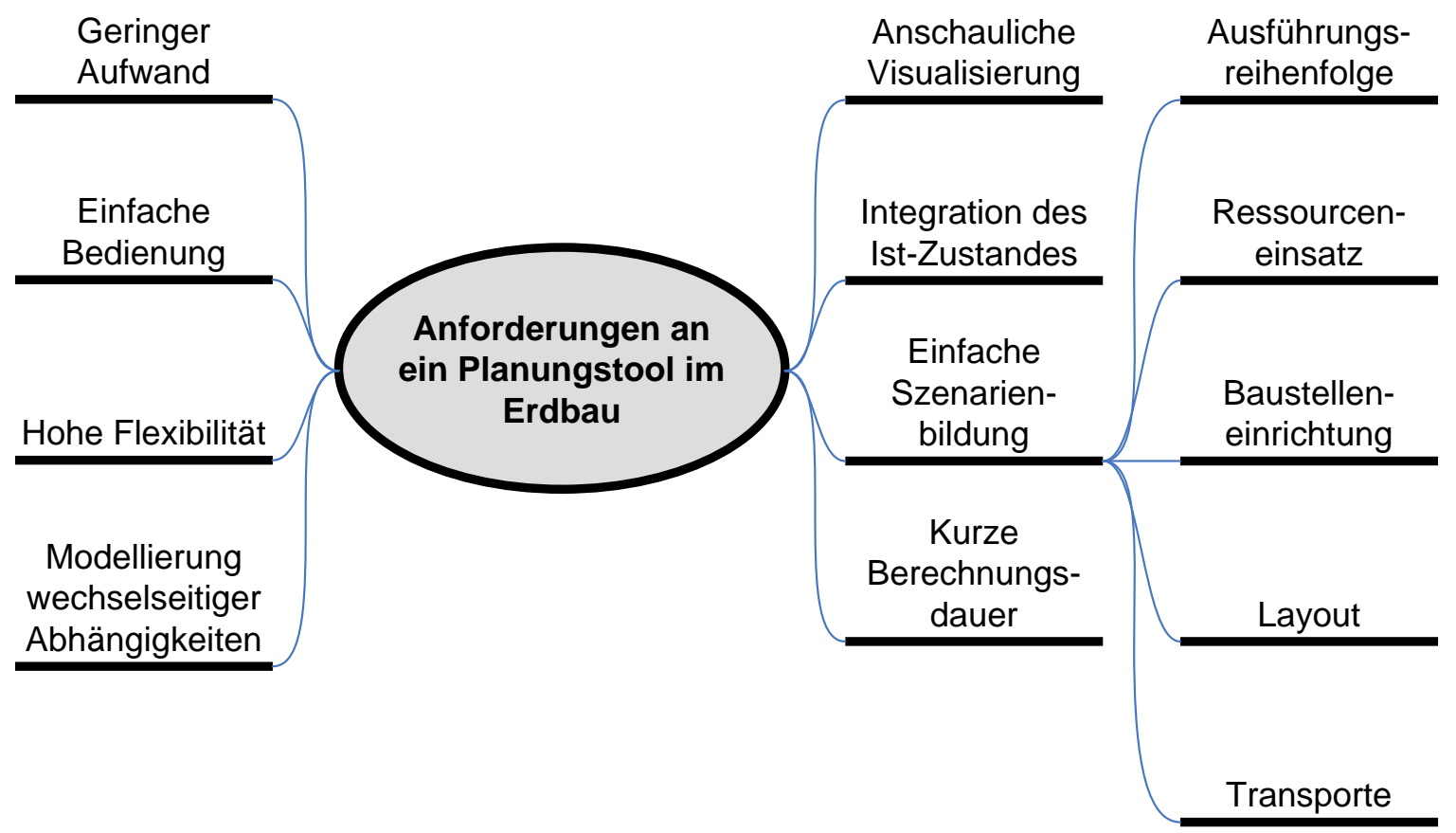

Abbildung 1: Anforderungen an ein Planungstool im Erdbau

Ein wichtiger Parameter ist hierbei die Baustelleneinrichtung, im Erdbau vor allem Lage und Ausbau der Baustraßen sowie Lage der Zwischenlager, Deponien und Materialquellen. Zudem sollten in der Simulation die Ausführungsreihenfolge der Erdbautätigkeiten sowie die Zuordnung der Ausbau- zu den Einbauorten veränderbar sein. Eine weitere Größe für die Szenarienbildung ist der Ressourceneinsatz. Die einzelnen Ressourcen sollten flexibel den jeweiligen Tätigkeiten zugeordnet werden können und in Art und Anzahl frei wählbar sein.

Eine detaillierte Modellierung der wechselseitigen Abhängigkeiten zwischen den Erdbauvorgängen muss zudem mit geringem Aufwand möglich sein, da sich die Abläufe mit jeder Baustelle ändern. Um immer ausgehend von der aktuellen Situation planen zu können, sollte der Ist-Zustand der Baustelle in das Planungstool integrierbar sein. Vor allem Änderungen im Ablauf müssen schnell eingepflegt werden können, da durch einen unerwarteten Baugrund oder durch Maschinenausfälle Umplanungen in Minuten bis Stunden getroffen werden müssen, um hohe Stillstandskosten $\mathrm{zu}$ vermeiden. Daher muss ein Tool zur Entscheidungsunterstützung vom verantwortlichen Bauleiter bedienbar sein und in diesem kurzen Zeitraum Ergebnisse liefern, welche durch eine anschauliche Visualisierung einfach interpretierbar sind.

Um diesen Anforderungen und Problemen der Ablaufplanung im Erdbau zu begegnen existieren bereits mehrere Ansätze, welche im nächsten Kapitel kurz erläutert werden.

\section{$3 \quad$ Stand der Technik und Forschung}

Die ereignisorientierte Ablaufsimulation ist in ihrer praktischen Anwendung im Bauwesen bislang wenig verbreitet. Vor allem im Hochbau werden jedoch (c) 2010 Logistics Journal : Proceedings - ISSN 2192-9084 
sogenannte 4D- bzw. 5D-Ablaufsimulationen eingesetzt. Diese Begriffe beschreiben im Bauwesen die Verknüpfung eines statischen Projektplans mit einem 3D-Modell. Durch Ein- und Ausblenden oder auch Einfärben von Bauteilen zu bestimmten Projektphasen ergibt sich daraus eine Visualisierung des Baufortschritts in 4D (3D + Zeit). Diesem Modell wird eine weitere Dimension hinzugefügt, indem die jeweils anfallenden Kosten der Tätigkeiten und Bauteile ebenfalls über den Projektzeitraum betrachtet werden [RIB10]. Durch eine reine Visualisierung können aber Engpässe oder Überschneidungen zwischen verschiedenen Tätigkeiten nicht aufgedeckt werden. Zudem wird nur die Zustandsänderung des Bauwerks sowie evtl. der Baustelleneinrichtung betrachtet, die wechselseitige Beeinflussung verschiedener Tätigkeiten kann nicht untersucht werden.

Eine Technik zur ereignisorientierten Modellierung von Baustellenabläufen sind PetriNetze. In diesen können z.B. zyklische Bauarbeiten einfach abgebildet werden [Fra99]. Die Komplexität der Modellierung erhöht sich jedoch stark mit der Anzahl der betrachteten Prozesse und deren Abhängigkeiten. Ein Beispiel für die Anwendung von erweiterten Petri-Netzen im Bauwesen ist die Arbeit von Chahrour, welche die Verbindung von CAD und Simulation von Erdbauprozessen auf Basis von PetriNetzen untersucht hat [Cha07].

Weiterhin wurden auf Basis von Aktivitätsdiagrammen mehrere bauspezifische Simulationssysteme konzipiert. Zwei dieser Systeme, CYCLONE und STROBOSCOPE, sind repräsentativ für das Spektrum dieser Modellierungs-Tools. CYCLONE ist ein gut etabliertes, weit verbreitetes und einfaches System, das leicht zu erlernen und effektiv für die Modellierung vieler einfacher Baumaßnahmen geeignet ist. STROBOSCOPE hingegen ist ein programmierbares und erweiterbares Simulationssystem zur Modellierung komplexer Baumaßnahmen, setzt aber dadurch eine längere Einarbeitungszeit und Programmierkenntnisse voraus [MI99].

Für den Bereich der Fabrikplanung bestehen bereits weit verbreitete Simulationssysteme zur Modellierung von Fertigungsabläufen (z.B. Enterprise Dynamics oder Plant Simulation). Diese umfangreichen, bereits in der Praxis erprobten Systeme bieten vorgefertigte Simulationsbausteine, die auf den Einsatz in intralogistischen Umgebungen abgestimmt sind. Durch den bausteinbasierten Aufbau wird ein Großteil des Modellierungs- und Implementierungsaufwands für die Simulationserstellung in eine projektunabhängige Phase vorverlagert. Die Erstellung eines neuen Simulationsmodells erfolgt anschließend über die graphische Verbindung und Parametrierung mehrerer Bausteine, so dass der Aufwand für das einzelne Simulationsprojekt erheblich sinkt. Ein weiterer Vorteil Systeme liegt in der Möglichkeit die simulierten Prozesse aus anschaulich zu visualisieren.

Daher werden diese Systeme auch im Baubereich verstärkt genutzt, obwohl deren Standardbausteine bisher nur für den Einsatz in Produktion und Intralogistik abgestimmt sind. Erste Anwendungen für die Simulation von Hochbauprozessen wurden bereits in der Forschung umgesetzt [KBSB07; Web07]. Ziel ist es nun die 
Vorteile der bausteinbasierten Modellierung auch in der Ablaufplanung von Erd- und Tiefbau nutzbar zu machen.

\section{$4 \quad$ Modellierung und Implementierung einer Bausteinbibliothek}

Aufgrund der vielfältigen Zielgrößen, sowie der veränderbaren Parameter und einzuhaltenden Randbedingungen ist die Flexibilität des Simulationssystems eine wichtige Eigenschaft für den Einsatz im Erdbau. Um dafür eine Grundlage zu schaffen, ist im Rahmen des Forschungsprojekts ForBAU eine Bausteinbibliothek für den Erd- und Tiefbau modelliert und in Plant Simulation von Siemens PLM implementiert worden. Die Bibliothek umfasst Bausteine für die Modellierung der baustellenbedingten Vorgänge, interne Verwaltungsbausteine, spezifische Objekte für die Baustelleneinrichtung sowie Funktionalitäten für den Datenim- und -export. Auf diese wird in den folgenden Abschnitten eingegangen.

\subsection{Schnittstellendefinition}

Wie bereits in der Anforderungsanalyse erwähnt, muss der Aufwand zur Erstellung eines Simulationsmodells gering gehalten werden. Die Einbettung der Simulation in den vorhandenen Planungsablauf sowie die Integration von vorhandenen Planungsinformationen aus anderen Systemen ist daher obligatorisch.

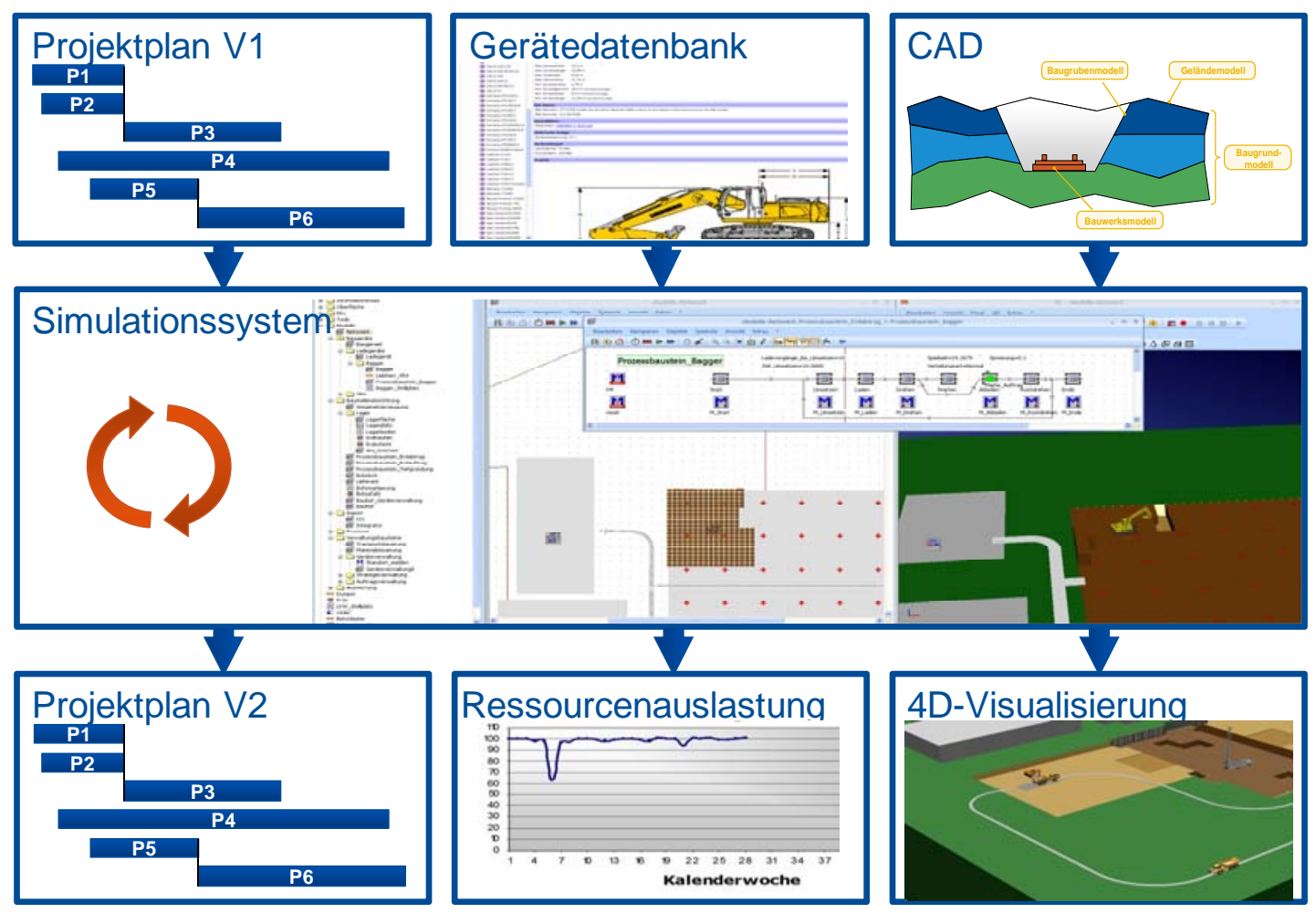

Abbildung 2: Schnittstellen der Simulation

In dem in Abbildung 2 dargestellten Schnittstellenkonzept sind die Ein- und Ausgangsdaten für die Ablaufsimulation dargestellt. Zunächst kann ein vorweg erstellter Projektplan aus einem konventionellen Projektmanagementtool (z.B. MS 
Project) in die Simulationsumgebung importiert werden. Relevant sind vor allem Informationen zu Beginn, Dauer und Ende der einzelnen Vorgänge sowie die für die Ausführung notwendigen Ressourcen und deren Einsatzzeiten. Der Projektplan wird im Simulationssystem in Prozesse übersetzt, welche ohne weitere Informationen ablauffähig sind die in der Simulation validiert und entsprechend den Ergebnissen angepasst werden. Schwer vorhersehbare Prozesse können hierbei im Detail geplant werden, um deren Abläufe spezifisch und ausführlich zu simulieren.

Maschinendaten werden aus einer Gerätedatenbank importiert. Zu diesem Zweck ist eine Schnittstelle für die Baumaschinendatenbank Equipment Information System (EIS) entwickelt worden [GKF ${ }^{+}$08]. EIS ermöglicht die einfache Auswahl geeigneter geeignete Maschinen durch umfangreiche Suchfunktionalitäten. Die spezifischen Eigenschaften der ausgewählten Maschinen können über die Schnittstelle direkt in die Simulationsumgebung importiert werden. Zudem werden die in der Datenbank hinterlegten 3D-Modelle der Baumaschinen für die Visualisierung verwendet.

Weitere wichtige Eingangsgrößen für die Simulation von Erdbauprozessen sind Volumen und Lage der auf- und abzutragenden Erdkörper. Meist wird deren Volumen mit dem Verfahren nach Gauß-Elling berechnet [DD10]. Dabei wird aus zwei Querprofilen eine gemittelte Fläche erstellt und diese mit dem Abstand zwischen den beiden Querprofilen multipliziert. Dieses Verfahren birgt vor allem bei gekrümmten Flächen, komplizierten Geometrien und große Abständen Ungenauigkeiten. Um die Massenermittlung zu erleichtern, wurde im Forschungsverbund ForBAU ein Tool geschaffen, mit dem das Untergrund-, das Oberflächen- sowie das Bauwerksmodell miteinander verschnitten und daraus das Volumen der jeweils abzutragenden und aufzubringenden Erdmassen bestimmt wird. Die Erdmassen werden in eine Vielzahl gleichförmiger Quader (Voxel) unterteilt, welche Informationen über Position, Volumen und Bodenart enthalten [JLOB08]. Die Voxelstruktur der ab- und der aufzutragenden Volumina wird anschließend über eine XML-Datei in die Simulationsumgebung importiert. Durch die feine Auflösung großer Erdkörper in kleine Voxel kann der Ablauf des Erdbaus sehr genau ermittelt und visualisiert werden. Neben den Erdmassen wird die Umgebung als 3D-Modell (JTFormat) importiert, um Baustraßen, Zufahrten, Lagerflächen, etc. räumlich planen zu können.

Als Ergebnis der Simulation entsteht ein verbesserter Projektplan, in dem auch feingranular aufgeteilte Vorgänge betrachtet werden können. Dieser Projektplan, dem die benötigten Ressourcen und Materialien hinterlegt sind, kann anschließend in der Ausführungsphase als Basis für die Bedarfsermittlung und die Steuerung der Anlieferungen verwendet werden. Die Auswertungen können zusätzlich für eine Prozesskostenrechnung genutzt werden, um detaillierte Kosteninformationen über die jeweils simulierten Szenarien zu erhalten. 


\subsection{Modellierung der Erdbauvorgänge}

Für die Modellierung der Erdbauvorgänge in der Simulation wurden bestehende Berechnungsverfahren [Bau07; Hüs92; Gir03] für die einzelnen Erd- und Tiefbauprozesse analysiert und hinsichtlich ihrer Eignung für den simulativen Einsatz überprüft. Im Erdbau werden fünf Teilvorgänge Lösen, Laden, Fördern, Einbauen und Verdichten unterschieden. Die ersten beiden Teilvorgänge Lösen und Laden werden in der Regel von einem Gerät, z.B. einem Bagger, übernommen. Die Berechnung dieser Teilvorgänge sowie der des Einbauens und Verdichtens ist ausreichend bekannt, durch vielfältige Einflussfaktoren parametrierbar und kann grundsätzlich für die Ablaufsimulation verwendet werden. Die entsprechenden Verfahren werden daher lediglich an die ereignisorientierte Modellierung angepasst und um statistische Komponenten erweitert.

Deutliches Optimierungspotenzial liegt hingegen in der Berechnung der Transportleistung, genauer in der Prognose von Umlaufzeiten spezifischer Transportfahrzeuge. Bisherige Verfahren berücksichtigen die dynamischen Bewegungen der Fahrzeuge nicht, Verzögerungs- und Beschleunigungszeiten sowie geringere Geschwindigkeiten bei Kurvenfahrten werden somit nicht betrachtet. Dies führt je nach Streckenprofil zu großen Abweichungen in den Fahrzeiten und somit zu maßgeblichen Ungenauigkeiten bei der Planung von Transportkapazitäten. Dabei sind die Transporte oft für einen Großteil der Erdbaukosten verantwortlich.

Um die Berechnung der Fahrzeiten zu verbessern, kann die Technik der kinematischen Simulation verwendet werden. Diese bietet die Möglichkeit für jedes Fahrzeug ein Geschwindigkeitsprofil je nach befahrener Strecke und aktuellem Beladungszustand $z u$ erstellen. Dabei wird in sehr kleinen Zeitschritten die Beschleunigungsfähigkeit des Fahrzeugs in Abhängigkeit der aktuellen Geschwindigkeit, den Fahrzeugeigenschaften und den Streckenparametern ermittelt. Ist die Antriebskraft im Vergleich zu den Fahrwiderständen zu klein, verringert sich die Geschwindigkeit in einem Zeitschritt, ansonsten wird sie erhöht. Grenzgeschwindigkeiten können sowohl für das Fahrzeug, als auch für die verschiedenen Streckenabschnitte angegeben werden, um beispielsweise Geschwindigkeitsbegrenzungen oder Staueinflüsse zu berücksichtigen. Durch das Verfahren der kinematischen Simulation wird es zudem innerhalb der Simulation möglich verschiedene Fahrzeuge miteinander zu vergleichen und damit eine optimale Maschinenkombination für den Erdbau zu finden. 


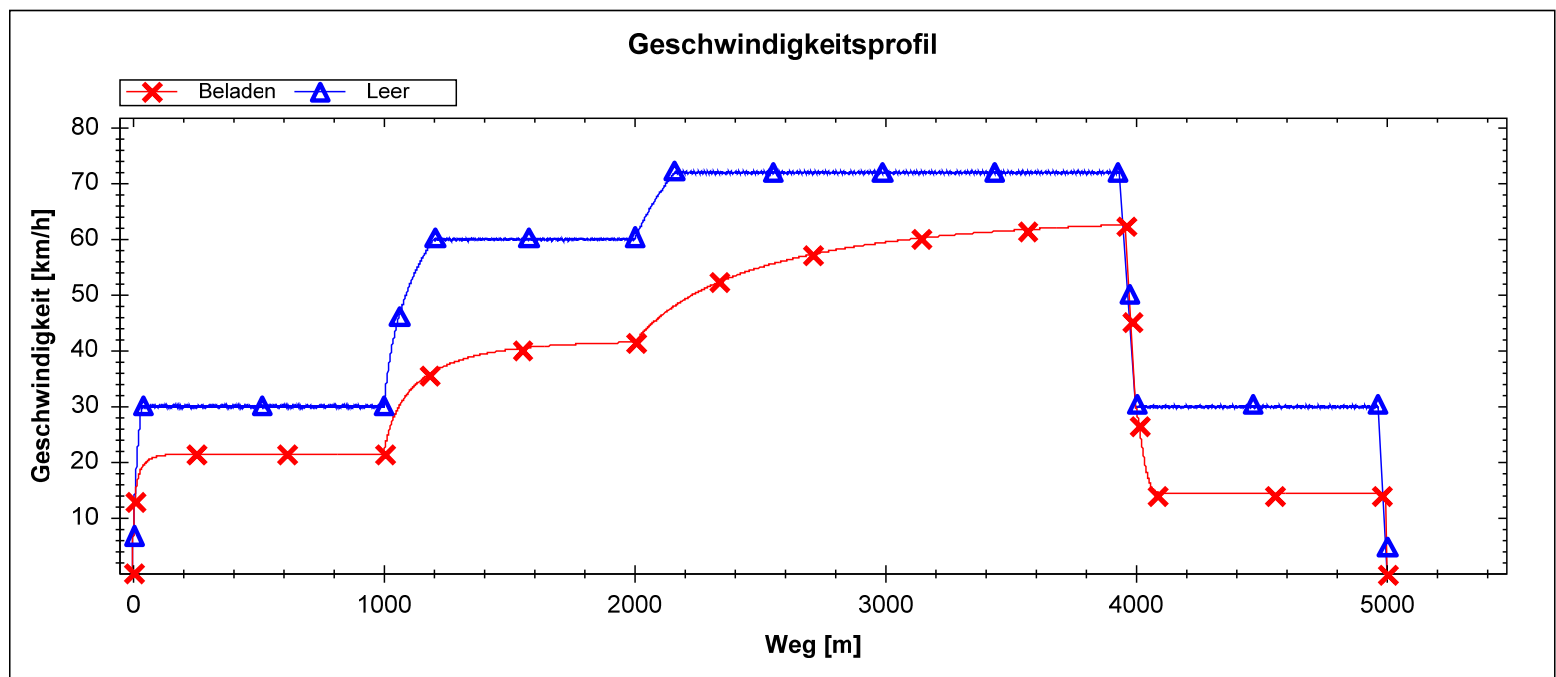

Abbildung 3: Simuliertes Geschwindigkeitsprofil

Ein Beispiel hierfür zeigt Abbildung 3. Im leeren Zustand erreicht das Fahrzeug die Grenzgeschwindigkeiten der Streckenabschnitte, im beladenen Zustand ist nur das Verhältnis von Fahrzeugleistung zu den Fahrwiderständen ausschlaggebend. Der vorgestellte Algorithmus wurde positiv getestet [GKFW09], so dass dieser in der Erdbausimulation für alle relevanten Transportvorgänge angewendet werden soll. Auf einer Baustelle bestehen meist mehrere alternative Stecken, auf denen die Transporte ausgeführt werden können, so dass zur Ermittlung des zeitlich kürzesten Weges ein Wegfindungsalgorithmus eingesetzt und mit der kinematischen Simulation gekoppelt werden muss.

Tabelle 1: Wegfindungsalgorithmen nach [KN05]

\begin{tabular}{|c|c|c|c|}
\hline Algorithmus & $\begin{array}{c}\text { Berechnung des kürzesten } \\
\text { Pfades zwischen }\end{array}$ & Rechenaufwand $^{2}$ & $\begin{array}{l}\text { heuris- } \\
\text { tisch }\end{array}$ \\
\hline$A^{*}$ & Startknoten und Zielknoten & $O(n \cdot \log n)$ & ja \\
\hline Dijkstra & Startknoten und Zielknoten & $O(n \cdot \log n+m)$ & nein \\
\hline Bellman-Ford & $\begin{array}{l}\text { Startknoten und allen } \\
\text { anderen Knoten }\end{array}$ & $O(n \cdot m)$ & nein \\
\hline Johnson & allen Knoten & $O\left(n \cdot m+n^{2} \log n\right)$ & nein \\
\hline Floyd-Warshall & allen Knoten & $O\left(n^{3}\right)$ & nein \\
\hline
\end{tabular}

In Tabelle 1 sind mehrere potenzielle Algorithmen mit ihren relevanten Eigenschaften zusammengefasst. Das wichtigste Bewertungskriterium ist in diesem Fall die Schnelligkeit des Algorithmus, da sich der Beladungszustand der Fahrzeuge sowie die Kennwerte der Streckenabschnitte je nach Witterung und Projektverlauf ändern und damit die Fahrzeitberechnung für jede Fahrt erneut durchgeführt werden muss.

\footnotetext{
${ }^{2}$ Berechnung über die Landauschen Symbole: Das Symbol $\mathrm{O}()$ gibt an, dass der Rechenaufwand für den Algorithmus maximal mit der in den Klammern angegebenen Funktion steigt. $n$ bezeichnet dabei die Anzahl der betrachteten Wegknoten, $m$ eine Teilmenge davon.
} 
Der jeweilige Rechenaufwand wird mit Hilfe der Landauschen Symbole angegeben. Diese verschleiern zwar die genaue Größe, sind aber für einen qualitativen Vergleich von Algorithmen im Allgemeinen aussagekräftig genug [Heu03]. Der Rechenaufwand kann aus Tabelle 1 entnommen werden. Diese verdeutlicht, dass mit steigender Wegknotenzahl der $A^{*}$-Algorithmus derjenige mit dem geringsten Rechenaufwand ist. Dieser hat allerdings für die Kombination mit der Kinematiksimulation den Nachteil, dass zur Verkürzung der Rechenzeit eine Schätzfunktion für den verbleibenden Weg zum Ziel (in der Regel die Luftlinie) verwendet wird. Da aber der kürzeste Weg auf Basis der Fahrzeit zu bestimmen ist, kann die Schätzfunktion der Luftlinie nicht angewendet werden, da für diese die Streckenparameter nicht bekannt sind, und somit keine Kinematiksimulation durchgeführt werden kann. Daher wurde für diesen Anwendungsfall der Dijkstra-Algorithmus ausgewählt und mit dem benutzten Kinematik-Algorithmus gekoppelt.

\subsection{Aufbau Bausteinbibliothek}

Die oben beschriebenen Algorithmen und Berechnungsverfahren wurden in verschiedenen Bausteinen in einer Bausteinbibliothek implementiert, um auch komplexe Abläufe im Erdbau abbilden zu können. Abbildung 4 zeigt den Gesamtzusammenhang der entwickelten Bausteine. Über Detaillierungsgrad und Simulationstiefe von Vorgängen wird bei der Erstellung des Simulationsmodells entschieden. Werden während der ausführlichen Experimente und Auswertungen kritische Prozesse identifiziert, kann die Detailstufe für diese Vorgänge erhöht werden.

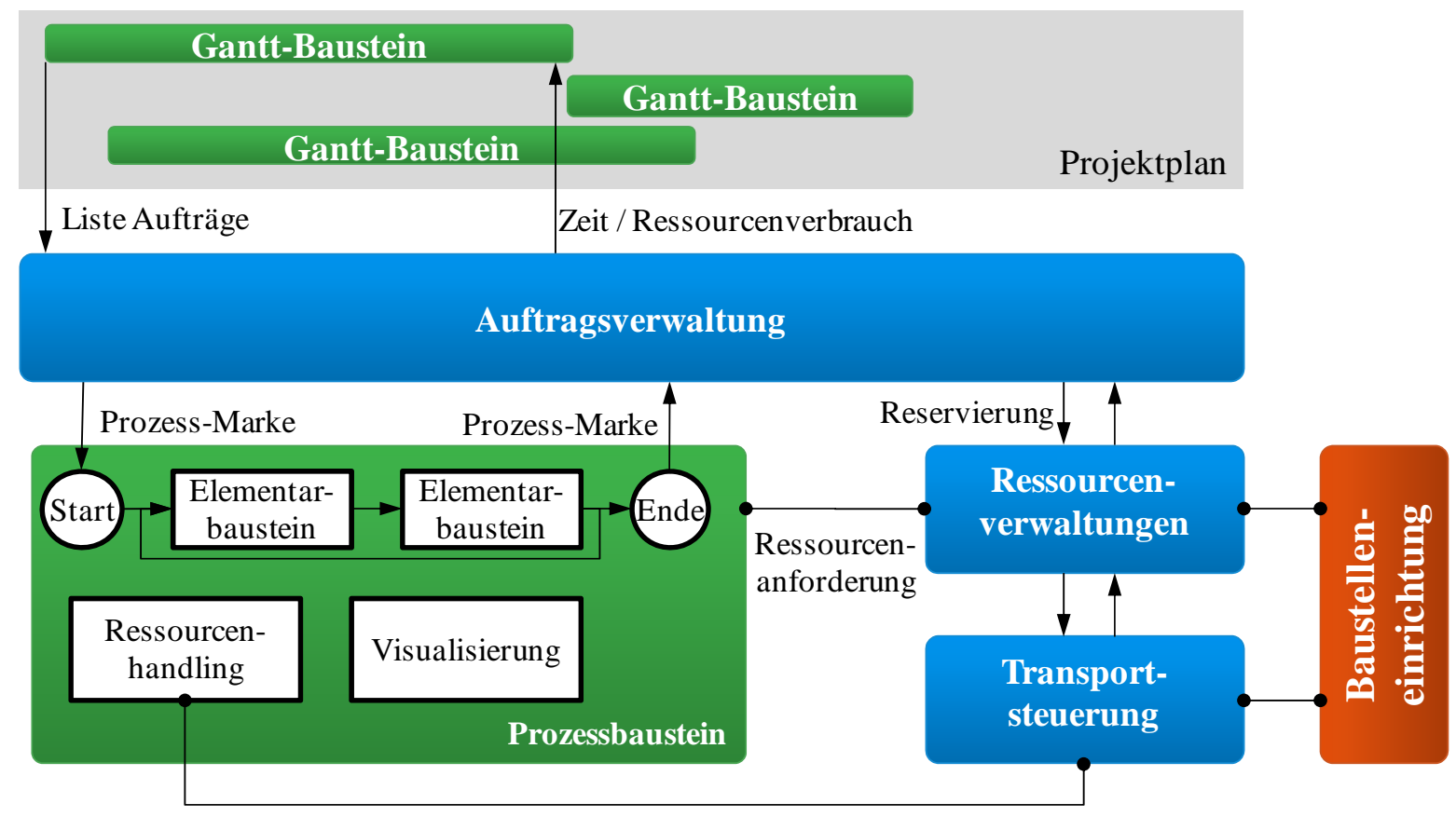

Abbildung 4: Interner Zusammenhang der Simulationsbausteine 
Die Vorgänge aus dem vorweg importierten Projektplan werden im Simulationssystem in ablauffähige Gantt-Bausteine übersetzt. Diese haben die Funktion, einen Vorgang in Bezug auf seine Vorgänger oder ab einem bestimmten Termin zu starten. Wird ein Vorgang nicht weiter unterteilt, ist der Simulation lediglich ein Zeitverbrauch hinterlegt, welcher bei Beginn und am Ende eine Zustandsänderung des Bauwerks auslösen kann. Letzteres dient neben der zeitlichen Einordnung der Prozesse auch zur Visualisierung des Ablaufs.

Für den Fall einer weiteren Detaillierung der Vorgänge werden die Informationen über die auszuführenden Teilprozesse vom jeweiligen Gantt-Baustein an eine Auftragsverwaltung übergeben. Dort werden die einzelnen Teilprozesse aus allen aktuell laufenden Vorgängen analog zum Constraint-basierten Simulationsansatz [KBSB07] geordnet. Hierbei wird in einer Auftragsverwaltung zum jeweiligen Simulationszeitpunkt abgefragt, welche anstehenden Tätigkeiten folgende Restriktionen einhalten:

- Alle vorausgehenden Tätigkeiten sind abgeschlossen.

- $\quad$ Alle nötigen Ressourcen (Personal, Geräte, Flächen, Material) stehen zur

Verfügung.

Ist eine Tätigkeit ausführbar, wird diese gestartet. Für die Überprüfung, ob eine Ressource für eine Tätigkeit grundsätzlich geeignet ist, sind Ressourcenverwaltungen entwickelt worden. Falls mehrere Ressourcen die Auswahlkriterien erfüllen, können verschiedene Strategien hinterlegt werden, um Einfluss auf die Zuweisung zu nehmen. Strategien sind beispielsweise die Ressourcenwahl nach kürzester Entfernung zum Arbeitsort oder nach geringster Auslastung.

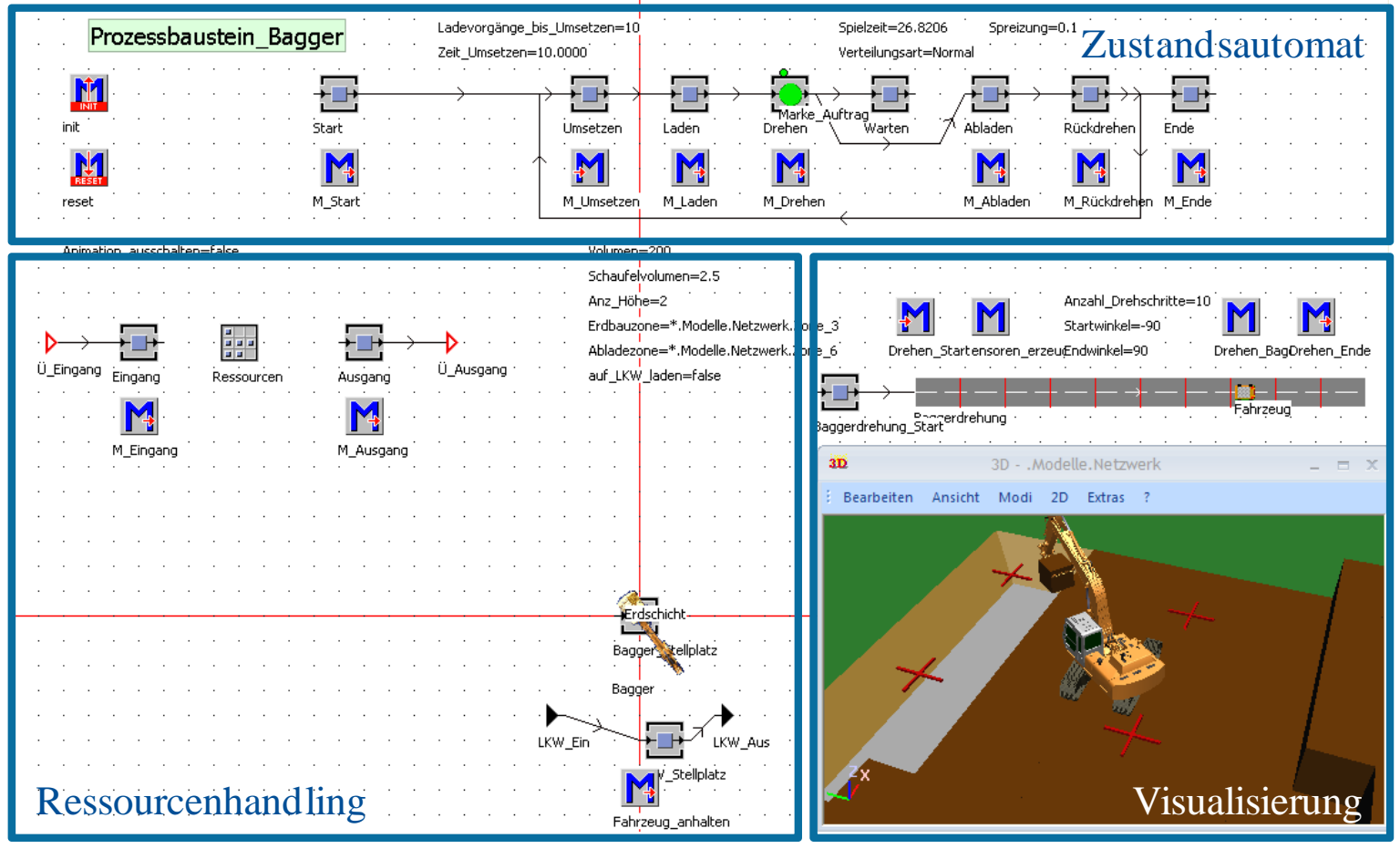

Abbildung 5: Beispiel eines Prozessbausteins 
Sind alle für einen Vorgang erforderlichen Ressourcen verfügbar, werden diese reserviert. Anschließend wird in der Auftragsverwaltung ein Prozessbaustein erzeugt, an den alle Informationen, die zum Ablauf der Tätigkeit notwendig sind, in Form einer Prozess-Marke übergeben werden. Abbildung 5 zeigt exemplarisch einen implementierten Prozessbaustein, welcher typischerweise in die drei Bereiche des Zustandsautomaten, des Ressourcenhandlings und der Visualisierung gegliedert ist. Im Zustandsautomaten (höchste Detaillierungsstufe) durchläuft die von der Auftragsverwaltung übergebene Marke nacheinander verschiedene Elementartätigkeiten, wobei jeweils das Eintreten und das Vollenden einer Tätigkeit einen neuen Zustand aktivieren. Einzelne Zustände können je nach Parametrierung des Prozessbausteins und den aktuellen Randbedingungen im Gesamtumfeld mehrfach durchlaufen, aber auch übersprungen werden. Für das Ressourcenhandling sind Elementartätigkeiten in Form von Elementarbausteinen umgesetzt, die jeweils Material, Personal, Geräte und Flächen buchen und wieder freigeben können, indem Anfragen an eine globale Ressourcensteuerung gestellt werden. Die angeforderte Ressource ist jeweils einem bestimmten Tätigkeitstyp zugeordnet. Die Steuerung ermittelt die entsprechend reservierte Ressource und übergibt diese an den Prozessbaustein. Sobald die Ressource am Auftragsort eingetroffen ist, wird der Prozess fortgesetzt und die Marke wird an die folgende Elementartätigkeit weitergeleitet. Des Weiteren übernimmt der Prozessbaustein Visualisierungsfunktionen wie Drehungen und Translationen von 2D- und 3D-Objekten, die den aktuellen Prozesszustand übersichtlich abbilden.

\section{$5 \quad$ Zusammenfassung}

Aufgrund der kurzen Planungszeiträume und dem hohen Aufwand wird bislang die ereignisorientierte Ablaufsimulationen in der Baupraxis kaum eingesetzt. Im Forschungsprojekt ForBAU wurde daher ein Konzept geschaffen, mit dem der Aufwand zur Erstellung einer Simulation durch eine bausteinbasierte Modellierung und die Wiederverwendung von vorhandenen Planungsdaten erheblich verringert wird. Dabei wurden bestehende Berechnungsgrundlagen für die Simulation angepasst und im Bereich der Transportmodellierung verfeinert. Durch die in Abbildung 4 dargestellte Bausteinstruktur sind die verschiedenen Vorgänge einer Baustelle, die Elemente der Baustelleneinrichtung sowie die verwendeten Ressourcen beliebig kombinierbar. Somit können verschiedene Szenarien mit wechselndem Maschineneinsatz und unterschiedlichen Randbedingungen vor Beginn einer Baumaßnahme gebildet werden. Durch den mehrstufig wählbaren Detaillierungsgrad ist es möglich, diejenigen Abläufe zu untersuchen, die als kritisch für den Gesamtprozess angesehen werden. Die Ablaufbausteine haben standardisierte Schnittstellen, so dass auch beliebige weitere Aktivitäten in der Bausteinbibliothek umgesetzt werden können. Zudem ist mit Simulationsbibliothek eine kombinierte 2D/3D-Visualisierung der Prozesse möglich, so dass diese auch als Kommunikationsmittel eingesetzt werden kann. 


\section{$6 \quad$ Literatur}

[Bau07] Bauer, H.: Baubetrieb. Berlin, Heidelberg: Springer, 2007.

[Cha07] Chahrour, R.: Integration von CAD und Simulation auf Basis von Produktmodellen im Erdbau. Kassel: Kassel Univ. Press, 2007.

[DD10] Deutsches Institut für Normung; Deutscher Vergabe- und Vertragsausschuss für Bauleistungen: VOB. Berlin: Beuth, Deutsches Institut für Normung; Deutscher Vergabe- und Vertragsausschuss für Bauleistungen, 2010.

[Fra99] Franz, V.: Simulation von Bauprozessen mit Hilfe von Petri-Netzen; In: Fortschritte in der Simulationstechnik. Weimar: 1999.

[Gir03] Girmscheid, G.: Leistungsermittlung für Baumaschinen und Bauprozesse. Berlin, u.a.: Springer, 2003

[GKF ${ }^{+}$08] Günthner, W. A.; Kessler, S.; Frenz, T.; Peters, B.; Walther, K.: Einsatz einer Baumaschinendatenbank (EIS) bei der Bayerischen BauAkademie; In: Tiefbau, Jahrgang 52 (2008)12, S. 736-738, 2008.

[GKFW09] Günthner, W. A.; Kessler, S.; Frenz, T.; Wimmer, J.: Transportlogistikplanung im Erdbau. München: Technische Universität München, 2009.

[Heu03] Heun, V.: Grundlegende Algorithmen. Wiesbaden: Vieweg, 2003.

[Hüs92] Hüster, F.: Leistungsberechnung der Baumaschinen. Düsseldorf: Werner, 1992.

[JLOB08] Ji, Y.; Lukas, K.; Obergriesser, M.; Borrmann, A.: Entwicklung integrierter 3D-Trassenproduktmodelle für die Bauablaufsimulation; In: Tagungsband des 20. Dresden: Forum Bauinformatik, 2008.

[KBSB07] König, M.; Beißert, U.; Steinhauer, D.; Bargstädt, H.-J.: ConstraintBased Simulation of Outfitting Processes in Shipbuilding and Civil Engineering; In: 6th EUROSIM Congress on Modeling and Simulation. Ljubljana, Slovenia: 2007.

[KN05] Krumke, S. O.; Noltemeier, H.: Graphentheoretische Konzepte und Algorithmen. Wiesbaden: Teubner, 2005.

[MI99] Martinez, J. C.; Ioannou, P. G.: General-Purpose Systems for Effective Construction Simulation (1999), S. Vol. 125, No. 4 (1999),265-276, 1999.

[RIB10] RIB Software AG: transparent; http://www.rib-software.com/de/ueberrib/transparent-das-magazin.html; Aufruf am 12.08.2010.

[Web07] Weber, J.: Simulation von Logistikprozessen auf Baustellen auf Basis von 3D-CAD Daten. Dortmund: Universität Dortmund, 2007. 